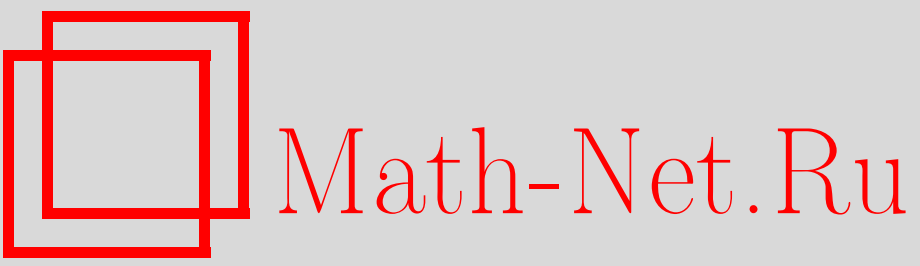

М. А. Спиряев, О некоторых свойствах стратегий Каги и Ренко для случайного блуждания, Теория вероятн. и ее примен., 2011, том 56, выпуск 2, 279-300

DOI: https://doi.org/10.4213/tvp4375

Использование Общероссийского математического портала Math-Net.Ru подразумевает, что вы прочитали и согласны с пользовательским соглашением

http://www.mathnet.ru/rus/agreement

Параметры загрузки:

IP : 54.224 .187 .69

26 апреля 2023 г., 18:31:53 


\title{
О НЕКОТОРЫХ СВОЙСТВАХ СТРАТЕГИЙ КАГИ И РЕНКО ДЛЯ СЛУЧАЙНОГО БЛУЖДАНИЯ
}

\begin{abstract}
В работе изучаются вероятностные характеристики методов технического анализа Каги (Kagi) и Ренко (Renko), используемых на финансовом рынке. Для случайного блуждания дается выражение для ожидаемой прибыли инвестора, использующего стратегию Каги, и исследуются его асимптотические свойства. В качестве промежуточных результатов установлены некоторые свойства, связанные с величинами «падения» и «размаха» случайного блуждания.
\end{abstract}

Ключевые слова и фразыл: случайное блуждание, величины «падения» и «размаха», стратегии Каги и Ренко, моменты Каги и Ренко.

1. Введение. На сегодняшний день существует множество графических методов технического анализа финансовых данных, позволяющих выявлять закономерности в движении цен и делать прогнозы путем анализа их графиков. Многие из этих методов посвящены проблеме выявления трендов и моментов, когда один тренд сменяется другим. Среди таких методов широкое распространение получили методы Каги (Kagi) и Ренко (Renko).

Происхождение методов Каги и Ренко связано с появившимися в Японии в 1870-х годах методами технического анализа, основанными на анализе колебаний цен. Впервые в литературе подробное описание методов Каги и Ренко, а также связанных с ними торговых стратегий было дано в работе [5]. В работе [2] проводится статистический анализ стратегий Каги и Ренко, а также приводится их математическая формализация.

Основная идея стратегий Каги и Ренко состоит в том, что решения о продаже/покупке актива принимаются лишь тогда, когда происходят существенные изменения цены. Этот подход позволяет акцентировать

\footnotetext{
${ }^{*}$ Московский государственный университет им. М.В. Ломоносова, механикоматематический факультет, Ленинские горы, 119991 Москва, Россия; e-mail: spiryaevmax@gmail.com

1) Работа поддержана проектами РФФИ 09-01-12157-офи-м, РФФИ 10-01-00518-а и SFB 701 при университете Билефельда.
} 
внимание трейдера только на значительных колебаниях цен и не рассматривать «шумы». Изучение свойств моментов Каги и Ренко, в которые принимаются решения, связано со свойствами процессов $\sup X-X$ и $\left|X-X_{0}\right|$, где $X$ является процессом цены. Эти моменты входят в выражение для прибыли инвестора, следующего стратегиям Каги и Ренко:

$$
\begin{aligned}
V_{\text {Kagi }} & =\sum_{m}(-1)^{m+1} \operatorname{sign}\left(X\left(\kappa_{0}\right)-X\left(\kappa_{0}^{*}\right)\right) \cdot\left(X\left(\kappa_{m}\right)-X\left(\kappa_{m-1}\right)\right), \\
V_{\text {Renko }} & =\sum_{m}(-1)^{m+1} \operatorname{sign}\left(X\left(\rho_{0}\right)-X\left(\rho_{0}^{*}\right)\right) \cdot\left(X\left(\rho_{m}\right)-X\left(\rho_{m-1}\right)\right),
\end{aligned}
$$

где $\kappa_{i}$ и $\rho_{i}$ являются моментами Каги и Ренко соответственно. Таким образом, для исследования ожидаемой прибыли инвестора необходимо определить конечномерные распределения процесса $X$ в моменты остановки Каги и Ренко:

$$
\operatorname{Law}\left(X\left(\kappa_{1}\right), \ldots, X\left(\kappa_{n}\right)\right), \quad \operatorname{Law}\left(X\left(\rho_{1}\right), \ldots, X\left(\rho_{n}\right)\right), \quad n \in \mathbf{N}
$$

В данной работе исследуется случай, когда процесс цены $X$ представляет собой случайное блуждание. Вычисляются конечномерные распределения процесса цены в моменты Каги и Ренко, преобразования Лапласа этих моментов, а также математическое ожидание прибыли инвестора, использующего стратегии Каги и Ренко.

2. Метод Каги. Для любой константы $H \in \mathbf{N}$ и случайного блуждания $(X(n))_{n \geqslant 0}$, определенного на вероятностном пространстве $\left(\Omega,\left(\mathscr{F}_{n}\right)_{n \geqslant 0}, \mathbf{P}\right)$, индуктивно определим последовательность моментов остановки $\left(\kappa_{i}, \kappa_{i}^{*}\right)_{i=0,1, \ldots}$.

(i) Базис: $\kappa_{0}=\inf \left\{u \geqslant 0: \max _{[0, u]} X-\min _{[0, u]} X=H\right\}$;

$$
\begin{aligned}
& \text { если } X\left(\kappa_{0}\right)=\max _{\left[0, \kappa_{0}\right]} X, \text { то } \kappa_{0}^{*}=\inf \left\{u \in\left[0, \kappa_{0}\right]: X(u)=\min _{\left[0, \kappa_{0}\right]} X\right\}, \\
& \text { если } X\left(\kappa_{0}\right)=\min _{\left[0, \kappa_{0}\right]} X, \text { то } \kappa_{0}^{*}=\inf \left\{u \in\left[0, \kappa_{0}\right]: X(u)=\max _{\left[0, \kappa_{0}\right]} X\right\} .
\end{aligned}
$$

(ii) $\amalg$ IIa $n \mapsto n+1$ :

$$
\begin{aligned}
& \text { если } X\left(\kappa_{n}\right)-X\left(\kappa_{n}^{*}\right)=H, \text { то } \\
& \kappa_{n+1}=\inf \left\{u \geqslant \kappa_{n}: \max _{\left[\kappa_{n}, u\right]} X-X(u)=H\right\}, \\
& \kappa_{n+1}^{*}=\inf \left\{u \in\left[\kappa_{n}, \kappa_{n+1}\right]: X(u)=\max _{\left[\kappa_{n}, \kappa_{n+1}\right]} X\right\} ; \\
& \text { если } X\left(\kappa_{n}\right)-X\left(\kappa_{n}^{*}\right)=-H, \text { то } \\
& \kappa_{n+1}=\inf \left\{u \geqslant \kappa_{n}: X(u)-\min _{\left[\kappa_{n}, u\right]} X=H\right\}, \\
& \kappa_{n+1}^{*}=\inf \left\{u \in\left[\kappa_{n}, \kappa_{n+1}\right]: X(u)=\min _{\left[\kappa_{n}, \kappa_{n+1}\right]} X\right\} .
\end{aligned}
$$


О п р е д е л е н и е 1 . Последовательность $\left(\kappa_{i}, \kappa_{i}^{*}\right)$ называется Kaгu$H$-конструкиией для процесса $X$. Моменты $\left(\kappa_{i}\right)_{i=0,1, \ldots}$ называются моментами остановки Каги.

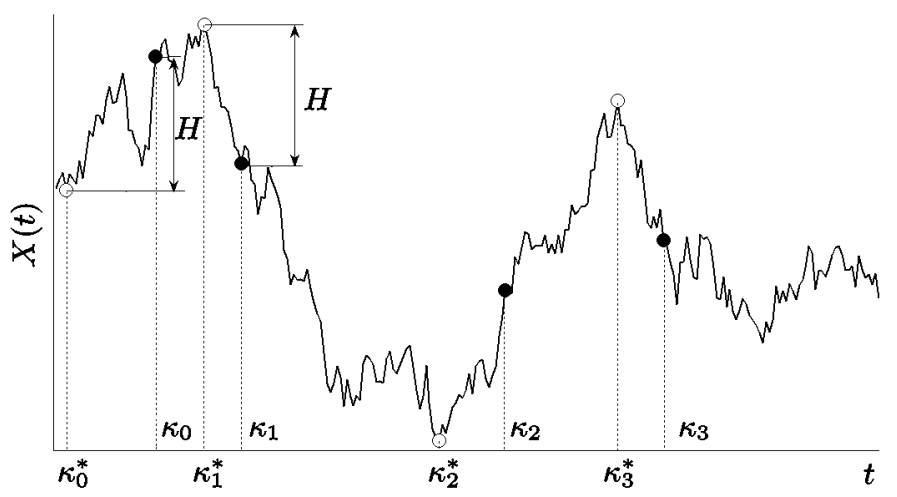

Рис. 1. Моменты остановки Каги.

2.1. Свойства моментов Каги для случайного блуждания.

Рассмотрим случайное блуждание $X=(X(n))_{n=0,1, \ldots}$ :

$$
X(n)=\sum_{i=1}^{n} \xi_{i}
$$

где $\left(\xi_{i}\right)_{i=1,2, \ldots}$ - независимые одинаково распределенные случайные величины, $\mathbf{P}\left(\xi_{i}=1\right)=p, \mathbf{P}\left(\xi_{i}=-1\right)=q$.

Теорема 1. Для произвольного $H \in \mathbf{N}$ случайное блуждание $X$ обладает следуюшими свойствами.

При $k_{0}>0$

$$
\begin{aligned}
\mathbf{P}\left(X\left(\kappa_{0}\right)\right. & \left.=k_{0}, \ldots, X\left(\kappa_{n}\right)=k_{n}\right) \\
& =C\left(k_{0}\right) \prod_{l=0}^{\lceil(n-1) / 2\rceil-1} p_{\min }\left(k_{2 l+2}-k_{2 l+1}\right) \prod_{l=0}^{\lceil(n-2) / 2\rceil} p_{\max }\left(k_{2 l+1}-k_{2 l}\right) .
\end{aligned}
$$

$\Pi p u k_{0}<0$

$$
\begin{aligned}
\mathbf{P}\left(X\left(\kappa_{0}\right)\right. & \left.=k_{0}, \ldots, X\left(\kappa_{n}\right)=k_{n}\right) \\
& =C\left(k_{0}\right) \prod_{l=0}^{\lceil(n-1) / 2\rceil-1} p_{\max }\left(k_{2 l+2}-k_{2 l+1}\right) \prod_{l=0}^{\lceil(n-2) / 2\rceil} p_{\min }\left(k_{2 l+1}-k_{2 l}\right) .
\end{aligned}
$$

Кроме того,

$$
\mathbf{E} \kappa_{n}=S_{0}+S_{\max }\left(P_{U}\left\lceil\frac{n}{2}\right\rceil+P_{D}\left\lceil\frac{n-1}{2}\right\rceil\right)
$$




$$
\begin{aligned}
& +S_{\min }\left(P_{U}\left\lceil\frac{n-1}{2}\right\rceil+P_{D}\left\lceil\frac{n}{2}\right\rceil\right) \quad \text { npu } p \neq q, \\
\mathbf{E} \kappa_{n}= & \left(\frac{1}{2}+n\right) H(H+1) \quad n p u p=q, \\
\mathbf{E} e^{-\lambda \kappa_{n}}= & F_{+}(H, \lambda)\left(D_{\max }\right)^{\lceil n / 2\rceil}\left(D_{\min }\right)^{\lceil(n-1) / 2\rceil} \\
& +F_{-}(H, \lambda)\left(D_{\max }\right)^{\lceil(n-1) / 2\rceil}\left(D_{\text {min }}\right)^{\lceil n / 2\rceil} .
\end{aligned}
$$

где $\lceil x\rceil=\min \{n \in \mathbf{Z}: n \geqslant x\}, k_{i} \in \mathbf{Z}$ и произведение $\prod_{l=0}^{-1} z_{l}$ мы полагаем равнылм 1. Определения величин $C, p_{\min }, p_{\max }, S_{0}, S_{\min }, S_{\max }, P_{U}, P_{D}$ даньи в (2.16), (2.18), (2.20), (2.26) и (2.23) соответственно. Определения величин $F_{ \pm}, D_{\max }$ и $D_{\min }$ даньь в (2.31) и (2.30).

2.2. Доказательство теоремы 1. Для доказательства теоремы 1 потребуются три леммы.

Лемма 1. Пусть $A \leqslant 0 \leqslant B$. Для случайного блуждания $X$ рассмотрим моменты выхода на уровни $A$ и $B$ :

$$
\tau^{A}=\inf \{n \geqslant 0: X(n)=A\}, \quad \tau^{B}=\inf \{n \geqslant 0: X(n)=B\},
$$

а также момент выхода прочесса $X$ из интервала $(A, B)$ :

$$
\tau^{[A, B]}=\tau^{A} \wedge \tau^{B} .
$$

Тогда для преобразования Лапласа момента $\tau^{[A, B]}$ справедливы следующие свойства:

$$
\begin{aligned}
& \mathbf{E} e^{-\lambda \tau^{[A, B]}} \mathbf{I}\left(\tau^{[A, B]}=\tau^{A}\right)=\frac{\operatorname{sh}\left(B\left(f_{+}(\lambda)+f_{-}(\lambda)\right) / 2\right) e^{A\left(f_{-}(\lambda)-f_{+}(\lambda)\right) / 2}}{\operatorname{sh}\left((B-A)\left(f_{+}(\lambda)+f_{-}(\lambda)\right) / 2\right)}, \\
& \mathbf{E} e^{-\lambda \tau^{[A, B]}} \mathbf{I}\left(\tau^{[A, B]}=\tau^{B}\right)=\frac{\operatorname{sh}\left(-A\left(f_{+}(\lambda)+f_{-}(\lambda)\right) / 2\right) e^{B\left(f_{-}(\lambda)-f_{+}(\lambda)\right) / 2}}{\operatorname{sh}\left((B-A)\left(f_{+}(\lambda)+f_{-}(\lambda)\right) / 2\right)},
\end{aligned}
$$

где

$$
f_{+}(\lambda)=\ln \frac{e^{\lambda}+\sqrt{e^{2 \lambda}-4 p q}}{2 p}, \quad f_{-}(\lambda)=\ln \frac{e^{\lambda}+\sqrt{e^{2 \lambda}-4 p q}}{2 q}, \quad \lambda \geqslant 0 .
$$

Д о к а з а т е л ь с т в о. Для произвольных $\mu_{1}, \mu_{2}>0$ рассмотрим два мартингала:

$$
\left(e^{\mu_{1} X(n)-n \ln g\left(\mu_{1}\right)}\right)_{n \geqslant 0}, \quad\left(e^{-\mu_{2} X(n)-n \ln g\left(-\mu_{2}\right)}\right)_{n \geqslant 0},
$$

где $g(\mu)=\mathbf{E} e^{\mu \xi_{1}}=p e^{\mu}+q e^{-\mu}$. Для любого $n \geqslant 0$ легко проверить, что

$$
0<e^{\mu_{1} X\left(\tau^{[A, B]} \wedge n\right)-\left(\tau^{[A, B]} \wedge n\right) \ln g\left(\mu_{1}\right)} \leqslant e^{\mu B}
$$


при условии, что $\ln g\left(\mu_{1}\right) \geqslant 0$. По теореме о преобразовании свободного выбора (см., например, [4]) и по теореме Лебега о мажорируемой сходимости получаем, что

$$
1=\lim _{n \rightarrow \infty} \mathbf{E} e^{\mu_{1} X\left(\tau^{[A, B]} \wedge n\right)-\left(\tau^{[A, B]} \wedge n\right) \ln g\left(\mu_{1}\right)}=\mathbf{E} e^{\mu_{1} X\left(\tau^{[A, B]}\right)-\tau^{[A, B]} \ln g\left(\mu_{1}\right)} .
$$

Последнее равенство справедливо в силу того, что $\mathbf{P}\left(\tau^{[A, B]}<\infty\right)=1$ (см. [3]). Аналогично показывается, что

$$
1=\lim _{n \rightarrow \infty} \mathbf{E} e^{-\mu_{2} X\left(\tau^{[A, B]} \wedge n\right)-\left(\tau^{[A, B]} \wedge n\right) \ln g\left(-\mu_{2}\right)}=\mathbf{E} e^{-\mu_{2} X\left(\tau^{[A, B]}\right)-\tau^{[A, B]} \ln g\left(-\mu_{2}\right)} .
$$

Из равенств (2.1) и (2.2) получаем следующую систему уравнений:

$$
\left\{\begin{array}{c}
e^{\mu_{1} A} \mathbf{E} e^{-\tau^{[A, B]} \ln g\left(\mu_{1}\right)} \mathbf{I}\left(\tau^{[A, B]}=\tau^{A}\right) \\
\quad+e^{\mu_{1} B} \mathbf{E} e^{-\tau^{[A, B]} \ln g\left(\mu_{1}\right)} \mathbf{I}\left(\tau^{[A, B]}=\tau^{B}\right)=1, \\
e^{-\mu_{2} A} \mathbf{E} e^{-\tau^{[A, B]} \ln g\left(-\mu_{2}\right)} \mathbf{I}\left(\tau^{[A, B]}=\tau^{A}\right) \\
+e^{-\mu_{2} B} \mathbf{E} e^{-\tau^{[A, B]} \ln g\left(-\mu_{2}\right)} \mathbf{I}\left(\tau^{[A, B]}=\tau^{B}\right)=1 .
\end{array}\right.
$$

Далее для любого $\lambda \geqslant 0$ выберем $\mu_{1}$ и $\mu_{2}$ так, чтобы выполнялись равенства

$$
\lambda=\ln g\left(\mu_{1}\right)=\ln g\left(-\mu_{2}\right) .
$$

Нетрудно показать, что система уравнений (2.4) имеет единственное решение, удовлетворяющее условиям $\lambda \geqslant 0, \ln \mu_{1} \geqslant 0, \ln \left(-\mu_{2}\right) \geqslant 0$ :

$$
\mu_{1}=\ln \frac{e^{\lambda}+\sqrt{e^{2 \lambda}-4 p q}}{2 p}=f_{+}(\lambda), \quad \mu_{2}=\ln \frac{e^{\lambda}+\sqrt{e^{2 \lambda}-4 p q}}{2 q}=f_{-}(\lambda) .
$$

Подставив $\mu_{1}$ и $\mu_{2}$ в (2.3), получаем систему из двух линейных уравнений:

$$
\left\{\begin{array}{rl}
e^{f_{+}(\lambda) A} \mathbf{E} & e^{-\tau^{[A, B]} \lambda} \mathbf{I}\left(\tau^{[A, B]}=\tau^{A}\right) \\
& +e^{f_{+}(\lambda) B} \mathbf{E} e^{-\tau^{[A, B]} \ln g(\lambda)} \mathbf{I}\left(\tau^{[A, B]}=\tau^{B}\right)=1, \\
e^{-f_{-}(\lambda) A} & \mathbf{E} e^{-\tau^{[A, B] \lambda)}} \mathbf{I}\left(\tau^{[A, B]}=\tau^{A}\right) \\
+ & e^{-f_{-}(\lambda) B} \mathbf{E} e^{-\tau^{[A, B]} \ln g(\lambda)} \mathbf{I}\left(\tau^{[A, B]}=\tau^{B}\right)=1 .
\end{array}\right.
$$

Решив эту систему, получаем утверждение леммы.

Следствие 1. Пусть $\beta^{[A, B]}$ и $\alpha^{[A, B]}$ обозначают вероятности выхода случайного блуждания $X$ из интервала $(A, B)$ через граничь $B$ и $A$ соответственно:

$$
\beta^{[A, B]}=\mathbf{P}\left(X\left(\tau^{[A, B]}\right)=B\right), \quad \alpha^{[A, B]}=\mathbf{P}\left(X\left(\tau^{[A, B]}\right)=A\right) .
$$


Тогда

$$
\begin{aligned}
\mathbf{E} e^{-\lambda \tau^{[A, B]}}= & \left(\operatorname{sh}\left(B \frac{f_{+}(\lambda)+f_{-}(\lambda)}{2}\right) e^{A\left(f_{-}(\lambda)-f_{+}(\lambda)\right) / 2}\right. \\
& \left.+\operatorname{sh}\left(-A \frac{f_{+}(\lambda)+f_{-}(\lambda)}{2}\right) e^{B\left(f_{-}(\lambda)-f_{+}(\lambda)\right) / 2}\right) \\
& \times\left[\operatorname{sh}\left((B-A) \frac{f_{+}(\lambda)+f_{-}(\lambda)}{2}\right)\right]^{-1} .
\end{aligned}
$$

$B$ cлучае $p \neq q$

$$
\begin{aligned}
\beta^{[A, B]}= & \frac{1-(q / p)^{A}}{(q / p)^{B}-(q / p)^{A}}, \quad \alpha^{[A, B]}=\frac{(q / p)^{B}-1}{(q / p)^{B}-(q / p)^{A}}, \\
& \mathbf{E} \tau^{[A, B]}=\frac{1}{p-q}\left(B \beta^{[A, B]}+A \alpha^{[A, B]}\right) .
\end{aligned}
$$

$B$ cлyчae $p=q$

$$
\beta^{[A, B]}=\frac{-A}{B-A}, \quad \alpha^{[A, B]}=\frac{B}{B-A}, \quad \mathbf{E} \tau^{[A, B]}=-A B .
$$

Другое, не основанное на лемме 1 , доказательство утверждений из следствия 1 можно прочитать, например, в [3].

Лемма 2. Пусть $H \in \mathbf{N}$. Для случайного блуждания $X$ рассмотрим моменты остановки

$$
\begin{aligned}
& \gamma_{\text {max }}=\inf \left\{k \geqslant 0: \max _{[0, k]} X-X(k)=H\right\}, \\
& \gamma_{\text {min }}=\inf \left\{k \geqslant 0: X(k)-\min _{[0, k]} X=H\right\} .
\end{aligned}
$$

Тогда преобразования Лапласа и математические ожидания моментов $\gamma_{\max } u \gamma_{\min }$ задаются следуюшим образом:

$$
\begin{aligned}
& \mathbf{E} e^{-\lambda \gamma_{\max }}=\operatorname{sh}\left(\frac{f_{+}(\lambda)+f_{-}(\lambda)}{2}\right) e^{-H\left(f_{-}(\lambda)-f_{+}(\lambda)\right) / 2} \\
& \times\left[\operatorname{sh}\left((H+1) \frac{f_{+}(\lambda)+f_{-}(\lambda)}{2}\right)-\operatorname{sh}\left(H \frac{f_{+}(\lambda)+f_{-}(\lambda)}{2}\right) e^{\left(f_{-}(\lambda)-f_{+}(\lambda)\right) / 2}\right]^{-1}, \\
& \mathbf{E} e^{-\lambda \gamma_{\min }}=\operatorname{sh}\left(\frac{f_{+}(\lambda)+f_{-}(\lambda)}{2}\right) e^{H\left(f_{-}(\lambda)-f_{+}(\lambda)\right) / 2} \\
& \times\left[\operatorname{sh}\left((H+1) \frac{f_{+}(\lambda)+f_{-}(\lambda)}{2}\right)-\operatorname{sh}\left(H \frac{f_{+}(\lambda)+f_{-}(\lambda)}{2}\right) e^{-\left(f_{-}(\lambda)-f_{+}(\lambda)\right) / 2}\right]^{-1},
\end{aligned}
$$

$$
\mathbf{E} \gamma_{\max }= \begin{cases}\frac{1}{p-q}\left(\frac{\beta^{[-H, 1]}}{\alpha^{[-H, 1]}}-H\right) & n p u p \neq q, \\ H(H+1) & n p u p=q,\end{cases}
$$




$$
\mathbf{E} \gamma_{\min }= \begin{cases}\frac{1}{p-q}\left(H-\frac{\alpha^{[-1, H]}}{\beta^{[-1, H]}}\right) & n p u p \neq q, \\ H(H+1) & \text { npu } p=q .\end{cases}
$$

Кроме того, распределения величин $\max _{\left[0, \gamma_{\max }\right]} X u \min _{\left[0, \gamma_{\min }\right]} X$ выцлядят следующим образом:

$$
\begin{gathered}
\mathbf{P}\left(\max _{\left[0, \gamma_{\max }\right]} X=k\right)=\left(\beta^{[-H, 1]}\right)^{k} \alpha^{[-H, 1]}, \quad k \geqslant 0, \\
\mathbf{P}\left(\min _{\left[0, \gamma_{\min }\right]} X=-k\right)=\left(\alpha^{[-1, H]}\right)^{k} \beta^{[-1, H]}, \quad k \geqslant 0,
\end{gathered}
$$

где $f_{+}(\lambda)=\ln \left(\left(e^{\lambda}+\sqrt{e^{2 \lambda}-4 p q}\right) /(2 p)\right), f_{-}(\lambda)=\ln \left(\left(e^{\lambda}+\sqrt{e^{2 \lambda}-4 p q}\right) /(2 q)\right)$, $\lambda \geqslant 0$. Величиньи $\alpha^{[A, B]}$ и $\beta^{[A, B]}$ определень в (2.6).

Д о к а з а т е л ь с т в о. Доказательство проведем только для величины $\gamma_{\max }$, поскольку доказательство для величины $\gamma_{\min }$ аналогично. Рассмотрим последовательность моментов остановки $\left(\delta_{n}\right)_{n \geqslant 0}$, которая задается следующим образом:

$$
\delta_{n}=\inf \left\{k \geqslant \delta_{n-1}: X(k)-X\left(\delta_{n-1}\right)=-H \text { или } 1\right\}, \quad \delta_{0}=0 .
$$

Также рассмотрим момент остановки $\sigma_{\max }$ :

$$
\sigma_{\max }=\inf \left\{k \geqslant 1: X\left(\delta_{k}\right)-X\left(\delta_{k-1}\right)=-H\right\} .
$$

Тогда $\gamma_{\max }$ можно представить в виде

$$
\gamma_{\max }=\delta_{\sigma_{\max }}=\sum_{k=1}^{\sigma_{\max }}\left(\delta_{k}-\delta_{k-1}\right) \quad \text { п.н. }
$$

Поскольку случайное блуждание $X$ обладает строго марковским свойством и имеет независимые приращения, то моменты $\left(\delta_{n}-\delta_{n-1}\right)_{n \geqslant 1}$ независимы и одинаково распределены, причем

$$
\delta_{n}-\delta_{n-1} \stackrel{\mathrm{d}}{=} \tau^{[-H, 1]}=\tau^{-H} \wedge \tau^{1} .
$$

Тогда из леммы 1 следует, что для любого $n \geqslant 1$

$$
\begin{gathered}
\mathbf{E} e^{-\lambda\left(\delta_{n}-\delta_{n-1}\right)} \mathbf{I}\left(X\left(\delta_{n}\right)-X\left(\delta_{n-1}\right)=-H\right) \\
=\frac{\operatorname{sh}\left(\left(f_{+}(\lambda)+f_{-}(\lambda)\right) / 2\right) e^{-H\left(f_{-}(\lambda)-f_{+}(\lambda)\right) / 2}}{\operatorname{sh}\left((H+1)\left(f_{+}(\lambda)+f_{-}(\lambda)\right) / 2\right)} \\
\mathbf{E} e^{-\lambda\left(\delta_{n}-\delta_{n-1}\right)} \mathbf{I}\left(X\left(\delta_{n}\right)-X\left(\delta_{n-1}\right)=1\right) \\
=\frac{\operatorname{sh}\left(H\left(\left(f_{+}(\lambda)+f_{-}(\lambda)\right) / 2\right)\right) e^{\left(f_{-}(\lambda)-f_{+}(\lambda)\right) / 2}}{\operatorname{sh}\left((H+1)\left(f_{+}(\lambda)+f_{-}(\lambda)\right) / 2\right)}
\end{gathered}
$$


где определения функций $f_{+}$и $f_{-}$даны в условии леммы. С помощью представления (2.9) и свойств случайного блуждания находим следующее выражение для преобразования Лапласа момента $\gamma_{\text {max }}$ :

$$
\begin{aligned}
& \mathbf{E} e^{-\lambda \gamma_{\max }}=\mathbf{E} \exp \left\{-\lambda \sum_{k=1}^{\sigma_{\max }}\left(\delta_{k}-\delta_{k-1}\right)\right\} \\
& =\sum_{n=1}^{\infty} \mathbf{E} \exp \left\{-\lambda \sum_{k=1}^{n}\left(\delta_{k}-\delta_{k-1}\right)\right\} \mathbf{I}\left(\sigma_{\max }=n\right) \\
& =\sum_{n=1}^{\infty} \mathbf{E} \exp \left\{-\lambda \sum_{k=1}^{n}\left(\delta_{k}-\delta_{k-1}\right)\right\} \mathbf{I}\left(X\left(\delta_{1}\right)=1\right) \\
& \times \mathbf{I}\left(X\left(\delta_{2}\right)-X\left(\delta_{1}\right)=1\right) \cdots \mathbf{I}\left(X\left(\delta_{n}\right)-X\left(\delta_{n-1}\right)=-H\right) \\
& =\sum_{n=1}^{\infty} \mathbf{E} \mathbf{E}\left(\exp \left\{-\lambda \sum_{k=1}^{n}\left(\delta_{k}-\delta_{k-1}\right)\right\} \mathbf{I}\left(X\left(\delta_{1}\right)=1\right)\right. \\
& \left.\times \mathbf{I}\left(X\left(\delta_{2}\right)-X\left(\delta_{1}\right)=1\right) \cdots \mathbf{I}\left(X\left(\delta_{n}\right)-X\left(\delta_{n-1}\right)=-H\right) \mid \mathscr{F}_{\delta_{n-1}}\right) \\
& =\sum_{n=1}^{\infty} \mathbf{E}\left(\exp \left\{-\lambda \sum_{k=1}^{n-1}\left(\delta_{k}-\delta_{k-1}\right)\right\}\right. \\
& \left.\times \mathbf{I}\left(X\left(\delta_{1}\right)=1, \ldots, X\left(\delta_{n-1}\right)-X\left(\delta_{n-2}\right)=1\right)\right) \mathbf{E} e^{-\lambda\left(\delta_{n}-\delta_{n-1}\right)} \\
& \times \mathbf{I}\left(X\left(\delta_{n}\right)-X\left(\delta_{n-1}\right)=-H\right) \\
& =\sum_{n=1}^{\infty} \mathbf{E}\left(\exp \left\{-\lambda \sum_{k=1}^{n-1}\left(\delta_{k}-\delta_{k-1}\right)\right\}\right. \\
& \left.\times \mathbf{I}\left(X\left(\delta_{1}\right)=1\right) \cdots \mathbf{I}\left(X\left(\delta_{n-1}\right)-X\left(\delta_{n-2}\right)=1\right)\right) \\
& \times \mathbf{E} e^{-\lambda \delta_{1}} \mathbf{I}\left(X\left(\delta_{1}\right)=-H\right) \\
& \cdots=\sum_{n=1}^{\infty}\left(\mathbf{E} e^{-\lambda \delta_{1}} \mathbf{I}\left(X\left(\delta_{1}\right)=1\right)\right)^{n-1} \mathbf{E} e^{-\lambda \delta_{1}} \mathbf{I}\left(X\left(\delta_{1}\right)=-H\right) \\
& =\frac{\mathbf{E} e^{-\lambda \delta_{1}} \mathbf{I}\left(X\left(\delta_{1}\right)=-H\right)}{1-\mathbf{E} e^{-\lambda \delta_{1}} \mathbf{I}\left(X\left(\delta_{1}\right)=1\right)} .
\end{aligned}
$$

Подставив выражения (2.11), получаем утверждение (2.7) леммы. Математическое ожидание момента $\gamma_{\max }$ можно получить либо непосредственно из (2.7), либо воспользовавшись тождеством Вальда:

$$
\mathbf{E} \gamma_{\max }=\mathbf{E}\left(\delta_{1}-\delta_{0}\right) \mathbf{E} \sigma_{\max } .
$$

При $p \neq q$ имеем:

$$
\mathbf{E} \gamma_{\max }=\frac{\beta^{[-H, 1]}-H \alpha^{[-H, 1]}}{(p-q) \alpha^{[-H, 1]}},
$$


поскольку

$$
\mathbf{E}\left(\delta_{n}-\delta_{n-1}\right)=\frac{1}{p-q}\left(\beta^{[-H, 1]}-H \alpha^{[-H, 1]}\right), \quad \mathbf{E} \sigma_{\max }=\frac{1}{\alpha^{[-H, 1]}} ;
$$

здесь первое равенство получено из (2.10) и следствия 1 , второе - из формулы для распределения момента $\sigma_{\max }$ :

$$
\begin{aligned}
\mathbf{P}\left(\sigma_{\max }=k\right) & =\mathbf{P}\left(X\left(\delta_{1}\right)=1, \ldots, X\left(\delta_{k-1}\right)=k-1, X\left(\delta_{k}\right)=k-1-H\right) \\
& =\left(\beta^{[-H, 1]}\right)^{k-1} \alpha^{[-H, 1]} .
\end{aligned}
$$

Случай $p=q$ рассматривается аналогично. Кроме того, из определения $\sigma_{\max }$ и $(2.12)$ получаем, что

$$
\mathbf{P}\left(\max _{\left[0, \gamma_{\max }\right]} X=k\right)=\mathbf{P}\left(\sigma_{\max }=k+1\right)=\left(\beta^{[-H, 1]}\right)^{k} \alpha^{[-H, 1]}
$$

Лемма 2 доказана.

Лемма 3. Пусть $H \in \mathbf{N}$. Для случайного блуждания $X$ рассмотрим введенный ранее момент остановки $\kappa_{0}$ :

$$
\kappa_{0}=\inf \left\{u \geqslant 0: \max _{[0, u]} X-\min _{[0, u]} X=H\right\} .
$$

Тогда распределение величины $X\left(\kappa_{0}\right)$ задается следующим образом:

$$
\mathbf{P}\left(X\left(\kappa_{0}\right)=k\right)= \begin{cases}\alpha^{[k-H, k]}-\alpha^{[k-H-1, k]} & \text { nрu } k=1,2, \ldots, H, \\ \beta^{[k, k+H]}-\beta^{[k, k+H+1]} & \text { nрu } k=-1,-2, \ldots,-H .\end{cases}
$$

Преобразование Лапласа момента остановки $\kappa_{0}$ задается следующим образом:

$$
\begin{aligned}
\mathbf{E} e^{-\lambda \kappa_{0}}= & \frac{\operatorname{sh}\left(\left(f_{+}(\lambda)+f_{-}(\lambda)\right) / 2\right)}{\operatorname{sh}\left(H\left(f_{+}(\lambda)+f_{-}(\lambda)\right) / 2\right) \operatorname{sh}\left((H+1)\left(f_{+}(\lambda)+f_{-}(\lambda)\right) / 2\right)} \\
& \times\left(G_{+}(H, \lambda)+G_{-}(H, \lambda)\right),
\end{aligned}
$$

где $f_{+}(\lambda)=\ln \left(\left(e^{\lambda}+\sqrt{e^{2 \lambda}-4 p q}\right) /(2 p)\right), f_{-}(\lambda)=\ln \left(\left(e^{\lambda}+\sqrt{e^{2 \lambda}-4 p q}\right) /(2 q)\right)$, $\lambda \geqslant 0 u$

$$
G_{ \pm}(H, \lambda)=\frac{\operatorname{ch}\left(f_{ \pm}(\lambda)(H+1 / 2)\right)-\operatorname{ch}\left(f_{ \pm}(\lambda) / 2\right)}{2 \operatorname{sh}\left(f_{ \pm}(\lambda) / 2\right)} .
$$

Д о к а з а т е ль с т в о. Возьмем произвольное $k$ такое, что $0<$ $k \leqslant H$, и рассмотрим момент $\tau^{(k-H, k)}$ первого выхода случайного блуждания $X$ из интервала $(k-H, k)$, а также момент $\chi_{k}$, задаваемый формулой

$$
\chi_{k}=\inf \left\{n \geqslant 0: X(n)-X\left(\tau^{[k-H, k]}\right)=-1 \text { или } H\right\} .
$$


Из определения момента $\kappa_{0}$ можно вывести следующее свойство:

$$
\mathbf{I}\left(X\left(\kappa_{0}\right)=k\right)=\mathbf{I}\left(X\left(\tau^{[k-H, k]}\right)=k-H\right) \mathbf{I}\left(X\left(\chi_{k}\right)-X\left(\tau^{[k-H, k]}\right)=H\right) .
$$

При этом в силу строго марковского свойства процесса $X$ и независимости приращений индикаторы, стоящие в правой части, независимы.

Следовательно, для $k=1, \ldots, H$

$$
\begin{aligned}
\mathbf{P}\left(X\left(\kappa_{0}\right)=k\right) & =\mathbf{P}\left(X\left(\tau^{[k-H, k]}\right)=k-H\right) \mathbf{P}\left(X\left(\chi_{k}\right)-X\left(\tau^{[k-H, k]}\right)=H\right) \\
& =\alpha^{[k-H, k]} \beta^{[-1, H]}=\alpha^{[k-H, k]}-\alpha^{[k-H-1, k]} .
\end{aligned}
$$

Заметим, что для $k>H$ непосредственно из определения момента $\kappa_{0}$ следует, что

$$
\mathbf{P}\left(X\left(\kappa_{0}\right)=k\right)=0, \quad k>H .
$$

Случай $k=-1, \ldots,-H$ разбирается аналогично.

Рассмотрим теперь преобразование Лапласа для момента $\kappa_{0}$ :

$$
\mathbf{E} e^{-\lambda \kappa_{0}}=\sum_{k=1}^{H} \mathbf{E} e^{-\lambda \kappa_{0}} \mathbf{I}\left(X\left(\kappa_{0}\right)=k\right)+\sum_{k=1}^{H} \mathbf{E} e^{-\lambda \kappa_{0}} \mathbf{I}\left(X\left(\kappa_{0}\right)=-k\right) .
$$

Используя свойство (2.13), получаем

$$
\begin{aligned}
& \mathbf{E} e^{-\lambda \kappa_{0}} \mathbf{I}\left(X\left(\kappa_{0}\right)=k\right) \\
& \quad=\mathbf{E}\left[e^{-\lambda \tau^{[k-H, k]}} \mathbf{I}\left(\tau^{[k-H, k]}=\tau^{k-H}\right)\right] \mathbf{E}\left[e^{-\lambda \tau^{[-1, H]}} \mathbf{I}\left(\tau^{[-1, H]}=\tau^{H}\right)\right], \\
& \mathbf{E} e^{-\lambda \kappa_{0}} \mathbf{I}\left(X\left(\kappa_{0}\right)=-k\right) \\
& \quad=\mathbf{E}\left[e^{-\lambda \tau^{[-k, H-k]}} \mathbf{I}\left(\tau^{[-k, H-k]}=\tau^{H-k}\right)\right] \mathbf{E}\left[e^{-\lambda \tau^{[-H, 1]}} \mathbf{I}\left(\tau^{[-H, 1]}=\tau^{-H}\right)\right] .
\end{aligned}
$$

Из леммы 1 следует, что

$$
\begin{aligned}
& \mathbf{E} e^{-\lambda \kappa_{0}} \mathbf{I}\left(X\left(\kappa_{0}\right)=k\right)=\frac{\operatorname{sh}\left(k \frac{f_{+}(\lambda)+f_{-}(\lambda)}{2}\right) \operatorname{sh}\left(\frac{f_{+}(\lambda)+f_{-}(\lambda)}{2}\right) e^{k \frac{f_{-}(\lambda)-f_{+}(\lambda)}{2}}}{\operatorname{sh}\left(H \frac{f_{+}(\lambda)+f_{-}(\lambda)}{2}\right) \operatorname{sh}\left((H+1) \frac{f_{+}(\lambda)+f_{-}(\lambda)}{2}\right)} \\
& \mathbf{E} e^{-\lambda \kappa_{0}} \mathbf{I}\left(X\left(\kappa_{0}\right)=-k\right)=\frac{\operatorname{sh}\left(k \frac{f_{+}(\lambda)+f_{-}(\lambda)}{2}\right) \operatorname{sh}\left(\frac{f_{+}(\lambda)+f_{-}(\lambda)}{2}\right) e^{-k \frac{f_{-}(\lambda)-f_{+}(\lambda)}{2}}}{\operatorname{sh}\left(H \frac{f_{+}(\lambda)+f_{-}(\lambda)}{2}\right) \operatorname{sh}\left((H+1) \frac{f_{+}(\lambda)+f_{-}(\lambda)}{2}\right)}
\end{aligned}
$$

Подставив эти выражения в (2.14), получим утверждение леммы.

Д о к а з а т е л ь с т в о т е о р е м ы 1 . Будем считать, что процесс $X$ задан на вероятностном пространстве $(\Omega, \mathscr{F}, \mathbf{P})$ с фильтрацией $\mathscr{F}_{k}^{X}=\sigma\{X(n), n \leqslant k\}$. Утверждение о распределении вектора $\left(X\left(\kappa_{0}\right), \ldots, X\left(\kappa_{n}\right)\right)$ будем доказывать по индукции.

Базис состоит в том, что

$$
\mathbf{P}\left(X\left(\kappa_{0}\right)=k_{0}\right)=C\left(k_{0}\right),
$$


где

$$
C\left(k_{0}\right)= \begin{cases}\left(1-\nu^{k_{0}}\right)\left(\frac{1}{1-\nu^{H \operatorname{sign}\left(k_{0}\right)}}-\frac{1}{1-\nu^{(H+1) \operatorname{sign}\left(k_{0}\right)}}\right) & \text { при } p \neq q, \\ \left|k_{0}\right|\left(\frac{1}{H}-\frac{1}{H+1}\right) & \text { при } p=q,\end{cases}
$$

$k_{0}= \pm 1, \ldots, \pm H$ и $\nu=p / q$. Данное утверждение непосредственно следует из леммы 3.

Шаг $(n-1) \mapsto n$. Без ограничения общности будем считать, что $k_{0}>0$ и $n$ четное, поскольку случай нечетного $n$ или отрицательного $k_{0}$ рассматривается аналогично. Заметим, что для произвольного случайного блуждания $S$ из леммы 2 следует, что

$$
\begin{aligned}
\mathbf{P}\left(S_{\gamma_{\max }}=r\right) & =\mathbf{P}\left(\max _{\left[0, \gamma_{\max }\right]} S=r+H\right) \\
& =\left(\beta^{[-H, 1]}\right)^{H+r} \alpha^{[-H, 1]}=p_{\max }(r), \\
\mathbf{P}\left(S_{\gamma_{\min }}=r\right) & =\mathbf{P}\left(\min _{\left[0, \gamma_{\min }\right]} S=r-H\right) \\
& =\left(\alpha^{[-1, H]}\right)^{H-r} \beta^{[-1, H]} \mathbf{I}(r \leqslant H)=p_{\min }(r),
\end{aligned}
$$

где

$$
\begin{aligned}
& p_{\max }(r)=\left(1-\frac{1}{\sum_{i=0}^{H} \nu^{i}}\right)^{H+r} \frac{1}{\sum_{i=0}^{H} \nu^{i}} \mathbf{I}(r \geqslant-H), \\
& p_{\min }(r)=\left(1-\frac{\nu^{H}}{\sum_{i=0}^{H} \nu^{i}}\right)^{H-r} \frac{\nu^{H}}{\sum_{i=0}^{H} \nu^{i}} \mathbf{I}(r \leqslant H) .
\end{aligned}
$$

Тогда, используя строго марковское свойство процесса $X$ и предположение индукции, получаем:

$$
\begin{aligned}
\mathbf{P}( & \left.X\left(\kappa_{0}\right)=k_{0}, \ldots, X\left(\kappa_{n}\right)=k_{n}\right) \\
= & \mathbf{P}\left(X\left(\kappa_{n}\right)=k_{n} \mid X\left(\kappa_{n-1}\right)=k_{n-1}, \ldots, X\left(\kappa_{0}\right)=k_{0}\right) \\
& \times \mathbf{P}\left(X\left(\kappa_{n-1}\right)=k_{n-1}, \ldots, X\left(\kappa_{0}\right)=k_{0}\right) \\
= & \mathbf{P}\left(X\left(\kappa_{n}\right)=k_{n} \mid X\left(\kappa_{n-1}\right)=k_{n-1}\right) \mathbf{P}\left(X\left(\kappa_{n-1}\right)=k_{n-1}, \ldots, X\left(\kappa_{0}\right)=k_{0}\right) \\
= & \mathbf{P}\left(X\left(\kappa_{n}\right)=k_{n} \mid X\left(\kappa_{n-1}\right)=k_{n-1}\right) C\left(k_{0}\right) \prod_{l=0}^{n / 2-2} p_{\min }\left(k_{n-2 l}-k_{n-2 l-1}\right) \\
& \times \prod_{l=0}^{n / 2-1} p_{\max }\left(k_{n-2 l-1}-k_{n-2 l-2}\right) .
\end{aligned}
$$

Вновь применяя строго марковское свойство, а также свойства (2.17), получаем, что для четного $n$ и положительного $k_{0}$

$$
\mathbf{P}\left(X\left(\kappa_{n}\right)=k_{n} \mid X\left(\kappa_{n-1}\right)=k_{n-1}\right)=p_{\min }\left(k_{n}-k_{n-1}\right) .
$$

Шаг индукции доказан. 
Для доказательства утверждения о математических ожиданиях моментов Каги также будем использовать метод математической индукции.

Несимметричный случай: $p \neq q$. Базис состоит в том, что

$$
\mathbf{E} \kappa_{0}=S_{0},
$$

где

$$
S_{0}=\frac{1}{p-q} \sum_{k=1}^{H} k \frac{\nu^{H+k+1}-2 \nu^{H+1}+2 \nu^{H}-\nu^{H+k}-\nu^{H-k}+\nu^{H-k+1}}{\left(1-\nu^{H}\right)\left(1-\nu^{H+1}\right)} .
$$

Чтобы это показать, рассмотрим мартингал $(X(k)-(p-q) k)_{k=0,1,2 \ldots}$. Из определения момента $\kappa_{0}$ несложно вывести следующее неравенство:

$$
\kappa_{0} \leqslant \gamma_{\max }+\gamma_{\min } \quad \text { п.н., }
$$

где моменты $\gamma_{\max }$ и $\gamma_{\min }$ определены в лемме 2. Отсюда получаем, что $\mathbf{E} \kappa_{0}<\infty$, и, применяя тождество Вальда, находим:

$$
\mathbf{E} X\left(\kappa_{0}\right)=(p-q) \mathbf{E} \kappa_{0} .
$$

Распределение величины $X\left(\kappa_{0}\right)$ известно из леммы 3 . Вычисляя отсюда математическое ожидание $X\left(\kappa_{0}\right)$, получаем $(2.19)$.

Шаг $(n-1) \mapsto n$. Будем считать, что $n$ является четным; случай нечетного $n$ рассматривается аналогично. Введем события

$$
\begin{aligned}
& U=\left\{\omega: X\left(\kappa_{0}\right)=\max _{\left[0, \kappa_{0}\right]} X\right\}=\left\{X\left(\kappa_{0}\right)>0\right\} \text { (восходящий тренд), } \\
& D=\left\{\omega: X\left(\kappa_{0}\right)=\min _{\left[0, \kappa_{0}\right]} X\right\}=\left\{X\left(\kappa_{0}\right)<0\right\} \text { (нисходящий тренд). }
\end{aligned}
$$

Обозначим вероятности событий $U$ и $D$ через $P_{U}$ и $P_{D}$ соответственно. Тогда из леммы 3 следует, что

$$
\begin{aligned}
P_{U}=\mathbf{P}\left(X\left(\kappa_{0}\right)>0\right) & =\sum_{k=1}^{H} \mathbf{P}\left(X\left(\kappa_{0}\right)=k\right) \\
& =\sum_{k=1}^{H} \frac{\nu^{H+k+1}-\nu^{H+1}+\nu^{H}-\nu^{H+k}}{\left(1-\nu^{H}\right)\left(1-\nu^{H+1}\right)}, \\
P_{D}=\mathbf{P}\left(X\left(\kappa_{0}\right)<0\right) & =\sum_{k=-H}^{-1} \mathbf{P}\left(X\left(\kappa_{0}\right)=k\right) \\
& =\sum_{k=1}^{H} \frac{-\nu^{H-k+1}+\nu^{H+1}-\nu^{H}+\nu^{H-k}}{\left(1-\nu^{H}\right)\left(1-\nu^{H+1}\right)} .
\end{aligned}
$$


Далее, рассмотрим следующие операторы на пространстве последовательностей $x=\left(x_{i}\right)_{i=1}^{\infty}$ :

$$
\begin{aligned}
& f_{\max }(x)=\min \left\{n: \max _{1 \leqslant i \leqslant n} x_{i}-x_{n}=H\right\}, \\
& f_{\min }(x)=\min \left\{n: x_{n}-\min _{1 \leqslant i \leqslant n} x_{i}=H\right\} .
\end{aligned}
$$

Тогда для момента $\kappa_{n}$ можно записать представление

$$
\begin{aligned}
\kappa_{n}= & \kappa_{n-1}+\mathbf{I}(U) f_{\min }\left(X\left(\kappa_{n-1}+1\right), X\left(\kappa_{n-1}+2\right), \ldots\right) \\
& +\mathbf{I}(D) f_{\max }\left(X\left(\kappa_{n-1}+1\right), X\left(\kappa_{n-1}+2\right), \ldots\right) .
\end{aligned}
$$

Взяв математическое ожидание от обеих частей этого равенства, получаем:

$$
\mathbf{E} \kappa_{n}=\mathbf{E} \kappa_{n-1}+P_{U} \frac{1}{p-q}\left(H-\frac{\alpha^{[-1, H]}}{\beta^{[-1, H]}}\right)+P_{D} \frac{1}{p-q}\left(\frac{\beta^{[-H, 1]}}{\alpha^{[-H, 1]}}-H\right),
$$

поскольку

$$
\begin{aligned}
& \mathbf{E}\left[\mathbf{I}(D) f_{\max }\left(X\left(\kappa_{n-1}+1\right), X\left(\kappa_{n-1}+2\right), \ldots\right)\right] \\
& =\mathbf{E}\left[\mathbf{I}(D) f_{\max }\left(X\left(\kappa_{n-1}+1\right)-X\left(\kappa_{n-1}\right), X\left(\kappa_{n-1}+2\right)-X\left(\kappa_{n-1}\right), \ldots\right)\right] \\
& =\mathbf{E}\left[\mathbf { E } \left(\mathbf { I } ( D ) f _ { \operatorname { m a x } } \left(X\left(\kappa_{n-1}+1\right)-X\left(\kappa_{n-1}\right),\right.\right.\right. \\
& \left.\left.\left.\quad X\left(\kappa_{n-1}+2\right)-X\left(\kappa_{n-1}\right), \ldots\right) \mid \mathscr{F}_{\kappa_{0}}\right)\right] \\
& =\mathbf{E}\left[\mathbf { I } ( D ) \mathbf { E } \left(f _ { \operatorname { m a x } } \left(X\left(\kappa_{n-1}+1\right)-X\left(\kappa_{n-1}\right),\right.\right.\right. \\
& =[\mathbf{E} \mathbf{I}(D)]\left[\mathbf{E}\left(f_{\max }\left(X\left(\kappa_{n-1}+2\right)-X\left(\kappa_{n-1}\right), \ldots\right) \mid \mathscr{F}_{\kappa_{0}}\right)\right] \\
& \left.\left.\quad X\left(\kappa_{n-1}+2\right)-X\left(\kappa_{n-1}\right), \ldots\right)\right] \\
& =\frac{1}{p-q}\left(\frac{\beta^{[-H, 1]}}{\alpha^{[-H, 1]}}-H\right) P_{D} .
\end{aligned}
$$

Здесь мы воспользовались тем, что оператор $f_{\max }$ инвариантен относительно сдвига, а величина $\mathbf{I}(D)$ измерима относительно $\mathscr{F}_{\kappa_{0}}$. Аналогично доказывается равенство для $f_{\min }$. Введем обозначения:

$$
\begin{aligned}
& S_{\max }=\frac{1}{p-q}\left(\frac{\beta^{[-H, 1]}}{\alpha^{[-H, 1]}}-H\right)=\frac{1}{p-q}\left(\frac{\nu-\nu^{H+1}}{1-\nu}-H\right), \\
& S_{\min }=\frac{1}{p-q}\left(H-\frac{\alpha^{[-1, H]}}{\beta^{[-1, H]}}\right)=\frac{1}{p-q}\left(H-\frac{1-\nu^{H}}{\nu^{H}-\nu^{H+1}}\right) .
\end{aligned}
$$

Тогда утверждение теоремы следует из (2.25) и предположения индукции. 
Симметричный случай: $p=q$. При $p=q$ событие $\left\{\kappa_{0}=k\right\}$ включает в себя траектории двух типов:

- траектории, для которых $\gamma_{\max }=k$,

- траектории, для которых $\gamma_{\min }=k$.

Между траекториями этих двух типов можно установить взаимно однозначное соответствие: если траекторию первого типа перевернуть (домножить на -1 ), то получится соответствующая траектория второго типа, и наоборот. Следовательно,

$$
\mathbf{E} \kappa_{0}=\frac{1}{2} \mathbf{E} \gamma_{\max }=\frac{1}{2} H(H+1), \quad P_{U}=P_{D}=\frac{1}{2} .
$$

Уравнение (2.25) в этом случае имеет вид

$$
\mathbf{E} \kappa_{n}=\mathbf{E} \kappa_{n-1}+H(H+1) \text {. }
$$

Отсюда получаем утверждение теоремы.

Для преобразования Лапласа момента $\kappa_{n}$, где $n$ - четное, рассмотрим следующее разложение:

$$
\mathbf{E} e^{-\lambda \kappa_{n}}=\mathbf{E} e^{-\lambda \kappa_{n}} \mathbf{I}(U)+\mathbf{E} e^{-\lambda \kappa_{n}} \mathbf{I}(D) .
$$

Воспользуемся свойством (2.24):

$$
\begin{aligned}
\mathbf{E} & e^{-\lambda \kappa_{n}} \mathbf{I}(U) \\
& =\mathbf{E} e^{-\lambda \kappa_{n-1}-\lambda f_{\min }\left(X\left(\kappa_{n-1}+1\right), X\left(\kappa_{n-1}+2\right), \ldots\right)} \mathbf{I}(U) \\
& =\mathbf{E}\left(\mathbf{E} e^{-\lambda \kappa_{n-1}-\lambda f_{\min }\left(X\left(\kappa_{n-1}+1\right), X\left(\kappa_{n-1}+2\right), \ldots\right)} \mathbf{I}(U) \mid \mathscr{F}_{\kappa_{n-1}}\right) \\
& =\mathbf{E}\left(\mathbf{E} e^{-\lambda \kappa_{n-1}-\lambda f_{\min }\left(X\left(\kappa_{n-1}+1\right)-X\left(\kappa_{n-1}\right), X\left(\kappa_{n-1}+2\right)-X\left(\kappa_{n-1}\right), \ldots\right)} \mathbf{I}(U) \mid \mathscr{F}_{\kappa_{n-1}}\right) \\
& =\left[\mathbf{E} e^{-\lambda \kappa_{n-1}} \mathbf{I}(U)\right]\left[\mathbf{E} e^{-\lambda f_{\min }\left(X\left(\kappa_{n-1}+1\right)-X\left(\kappa_{n-1}\right), X\left(\kappa_{n-1}+2\right)-X\left(\kappa_{n-1}\right), \ldots\right)}\right] . \quad(2.28)
\end{aligned}
$$

По лемме 2

$$
\begin{aligned}
& \mathbf{E} e^{-\lambda f_{\min }\left(X\left(\kappa_{n-1}+1\right)-X\left(\kappa_{n-1}\right), \ldots\right)} \\
& =\frac{\operatorname{sh}\left(H\left(f_{+}(\lambda)+f_{-}(\lambda)\right) / 2\right) e^{\left(f_{-}(\lambda)-f_{+}(\lambda)\right) / 2}}{\operatorname{sh}\left((H+1)\left(f_{+}(\lambda)+f_{-}(\lambda)\right) / 2\right)-\operatorname{sh}\left(\left(f_{+}(\lambda)+f_{-}(\lambda)\right) / 2\right) e^{H\left(f_{-}(\lambda)-f_{+}(\lambda)\right) / 2}} .
\end{aligned}
$$

Подставляя это равенство в (2.28) и обобщая полученные результаты на случай произвольного $n$, находим, что

$$
\begin{aligned}
& \mathbf{E} e^{-\lambda \kappa_{n}} \mathbf{I}(U)=\mathbf{E} e^{-\lambda \kappa_{0}} \mathbf{I}(U)\left(D_{\max }\right)^{\lceil n / 2\rceil}\left(D_{\min }\right)^{\lceil(n-1) / 2\rceil}, \\
& \mathbf{E} e^{-\lambda \kappa_{n}} \mathbf{I}(D)=\mathbf{E} e^{-\lambda \kappa_{0}} \mathbf{I}(D)\left(D_{\max }\right)^{\lceil(n-1) / 2\rceil}\left(D_{\min }\right)^{\lceil n / 2\rceil},
\end{aligned}
$$


где

$$
\begin{aligned}
& D_{\max }=\operatorname{sh}\left(\frac{f_{+}(\lambda)+f_{-}(\lambda)}{2}\right) e^{-H\left(f_{-}(\lambda)-f_{+}(\lambda)\right) / 2} \\
& \times\left[\operatorname{sh}\left((H+1) \frac{f_{+}(\lambda)+f_{-}(\lambda)}{2}\right)-\operatorname{sh}\left(H \frac{f_{+}(\lambda)+f_{-}(\lambda)}{2}\right) e^{\left(f_{-}(\lambda)-f_{+}(\lambda)\right) / 2}\right]^{-1} \\
& D_{\min }=\operatorname{sh}\left(\frac{f_{+}(\lambda)+f_{-}(\lambda)}{2}\right) e^{H\left(f_{-}(\lambda)-f_{+}(\lambda)\right) / 2} \\
& \times\left[\operatorname{sh}\left((H+1) \frac{f_{+}(\lambda)+f_{-}(\lambda)}{2}\right)-\operatorname{sh}\left(H \frac{f_{+}(\lambda)+f_{-}(\lambda)}{2}\right) e^{-\left(f_{-}(\lambda)-f_{+}(\lambda)\right) / 2}\right]^{-1}
\end{aligned}
$$

и $f_{+}(\lambda)=\ln \left(\left(e^{\lambda}+\sqrt{e^{2 \lambda}-4 p q}\right) /(2 p)\right), f_{-}(\lambda)=\ln \left(\left(e^{\lambda}+\sqrt{e^{2 \lambda}-4 p q}\right) /(2 q)\right)$.

$\mathrm{C}$ помощью представлений (2.15) можно показать, что

$$
\mathbf{E} e^{-\lambda \kappa_{0}} \mathbf{I}(U)=F_{+}(H, \lambda), \quad \mathbf{E} e^{-\lambda \kappa_{0}} \mathbf{I}(D)=F_{-}(H, \lambda),
$$

где

$$
\begin{aligned}
F_{ \pm}(H, \lambda)= & \frac{\operatorname{sh}\left(\left(f_{+}(\lambda)+f_{-}(\lambda)\right) / 2\right)}{\operatorname{sh}\left(H\left(f_{+}(\lambda)+f_{-}(\lambda)\right) / 2\right) \operatorname{sh}\left((H+1)\left(f_{+}(\lambda)+f_{-}(\lambda)\right) / 2\right)} \\
& \times\left(\frac{e^{f_{\mp}(\lambda)}}{2} \frac{1-e^{H f_{\mp}(\lambda)}}{1-e^{f_{\mp}(\lambda)}}-\frac{e^{-f_{ \pm}(\lambda)}}{2} \frac{1-e^{-H f_{ \pm}(\lambda)}}{1-e^{-f_{ \pm}(\lambda)}}\right) .
\end{aligned}
$$

Соединяя вместе $(2.27),(2.29),(2.30)$ и (2.31), получаем утверждение теоремы.

\section{3. Метод Ренко.}

3.1. Построение. Для случайного блуждания $(X(n))_{n \geqslant 0}$ и константы $H \in \mathbf{N}$ индуктивно определим последовательность моментов остановки $\left(\widetilde{\rho}_{i}\right)_{i=0,1, \ldots}$ :

$$
\widetilde{\rho}_{0}=0, \quad \ldots, \quad \widetilde{\rho}_{i+1}=\min \left\{u \geqslant \widetilde{\rho}_{i}:\left|X(u)-X\left(\widetilde{\rho}_{i}\right)\right|=H\right\} .
$$

Построим процесс $\tilde{X}=(\tilde{X}(n))_{n \geqslant 0}$ следующим образом:

$$
\tilde{X}(n)=X\left(\widetilde{\rho}_{i}\right), \quad n \in\left[\widetilde{\rho}_{i}, \widetilde{\rho}_{i+1}\right)
$$

Для процесса $\widetilde{X}$ построим его Каги- $H$-конструкцию $\left(\rho_{i}, \rho_{i}^{*}\right)_{i=0,1, \ldots}$.

О п р е д е л е н и е 2 . Последовательность $\left(\rho_{i}, \rho_{i}^{*}\right)_{i=0,1, \ldots}$ называется Ренко- $H$-конструкиией для процесса $X$. Моменты $\left(\rho_{i}\right)_{i=0,1, \ldots}$ называются моментами остановки Ренко. 


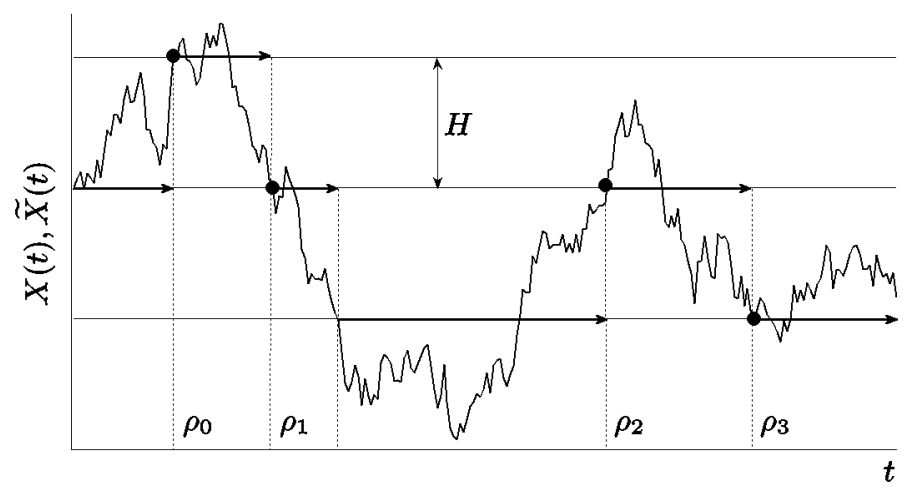

Рис. 2. Моменты остановки Ренко.

3.2. Свойства моментов Ренко для случайного блуждания.

Теорема 2. Для произвольного $H \in \mathbf{N}$ случайное блуждание $X$ обладает следуюшими свойствами.

При $r_{0}>0$

$$
\begin{aligned}
\mathbf{P}\left(X\left(\rho_{0}\right)=r_{0} H, \ldots, X\left(\rho_{n}\right)=r_{n} H\right)= & C\left(r_{0}\right) \prod_{l=0}^{\lceil(n-1) / 2\rceil-1} p_{\min }\left(r_{2 l+2}-r_{2 l+1}\right) \\
& \times \prod_{l=0}^{\lceil(n-2) / 2\rceil} p_{\max }\left(r_{2 l+1}-r_{2 l}\right) .
\end{aligned}
$$

При $r_{0}<0$

$$
\begin{aligned}
\mathbf{P}\left(X\left(\rho_{0}\right)=r_{0} H, \ldots, X\left(\rho_{n}\right)=r_{n} H\right)= & C\left(r_{0}\right) \prod_{l=0}^{\lceil(n-1) / 2\rceil-1} p_{\max }\left(r_{2 l+2}-r_{2 l+1}\right) \\
& \times \prod_{l=0}^{\lceil(n-2) / 2\rceil} p_{\min }\left(r_{2 l+1}-r_{2 l}\right),
\end{aligned}
$$

Кроме того,

$$
\begin{aligned}
\mathbf{E} \rho_{n}= & S_{0}+S_{\max }\left(P_{U}\lceil n / 2\rceil+P_{D}\left\lceil\frac{n-1}{2}\right\rceil\right) \\
& +S_{\min }\left(P_{U}\left\lceil\frac{n-1}{2}\right\rceil+P_{D}\left\lceil\frac{n}{2}\right\rceil\right) \quad n p u p \neq q, \\
\mathbf{E} \rho_{n}= & (2 n+1) H^{2} \quad n p u p=q, \\
\mathbf{E} e^{-\lambda \rho_{n}}= & F_{+}(H, \lambda)\left(D_{\max }\right)^{\lceil n / 2\rceil}\left(D_{\min }\right)^{\lceil(n-1) / 2\rceil} \\
& +F_{-}(H, \lambda)\left(D_{\max }\right)^{\lceil(n-1) / 2\rceil}\left(D_{\min }\right)^{\lceil n / 2\rceil},
\end{aligned}
$$

где $\lceil x\rceil=\min \{n \in \mathbf{Z}: n \geqslant x\}, k_{i} \in \mathbf{Z}$ и произведение $\prod_{l=0}^{-1} z_{l}$ мы полагаем равнылм 1. Определения величин $C, p_{\min }, p_{\max }, S_{0}, S_{\min }, S_{\max }, P_{U}, P_{D}$ 
будут даны в (3.3), (3.4), (3.5) и (3.6) соответственно. Определения величин $F_{ \pm}, D_{\max }$ и $D_{\min }$ будут даньв в (3.12), (3.9) и (3.10).

3.3. Доказательство теоремы 2. Для доказательства первых двух утверждений рассмотрим введенный ранее процесс $\widetilde{X}$. Этот процесс, определенный на прежнем вероятностном пространстве $(\Omega, \mathscr{F}, \mathbf{P})$, но с другой фильтрацией $\mathscr{F}_{n}=\sigma\left\{X\left(\widetilde{\rho}_{k}\right), k \leqslant n\right\}$, является случайным блужданием, умноженным на $H$ :

$$
\widetilde{X}(n)=\sum_{i=1}^{n} \eta_{i}
$$

где

$$
\mathbf{P}\left(\eta_{i}=H\right)=\beta^{[-H, H]}=\frac{\nu^{H}}{1+\nu^{H}}, \quad \mathbf{P}\left(\eta_{i}=-H\right)=\alpha^{[-H, H]}=\frac{1}{1+\nu^{H}} .
$$

Для процесса $\tilde{X}$ рассмотрим соответствующую ему последовательность $\widetilde{\kappa}_{i}$ моментов Каги с уровнем $\widetilde{H}=1$. Необходимо отметить, что случайные блуждания $X$ и $\widetilde{X}$ имеют разную временную шкалу, поскольку время, за которое процесс $\widetilde{X}$ делает $i$-й шаг, равно $\widetilde{\rho}_{i}$. При этом «формулы перехода» между характеристиками процессов $X$ и $\widetilde{X}$ имеют вид

$$
\begin{aligned}
& \mathbf{P}\left(X\left(\rho_{0}\right)=r_{0} H, \ldots, X\left(\rho_{n}\right)=r_{n} H\right) \\
& =\mathbf{P}\left(\widetilde{X}\left(\widetilde{\kappa}_{0}\right)=r_{0}, \ldots, \widetilde{X}\left(\widetilde{\kappa}_{n}\right)=r_{n}\right), \\
& \mathbf{E} \rho_{n}=\mathbf{E} \widetilde{\kappa}_{n} \mathbf{E} \tau^{[-H, H]},
\end{aligned}
$$

где $\tau^{[-H, H]}=\tau^{-H} \wedge \tau^{H}-$ момент выхода процесса $X$ из интервала $(-H, H)$. Здесь первое равенство выводится непосредственно из определения моментов $\rho_{k}$ и процесса $\widetilde{X}$. Второе равенство выводится из тождества Вальда и следующего свойства моментов Ренко:

$$
\rho_{n}=\sum_{i=1}^{\tilde{\kappa}_{n}} \widetilde{\rho}_{i} \quad \text { п.н., } \quad n \geqslant 0 .
$$

Характеристики процесса $\widetilde{X}$ в правой части (3.1) находятся из теоремы 1 , если в нее в качестве $\widetilde{H}$ и $\widetilde{\nu}$ подставить

$$
\widetilde{H}=1, \quad \widetilde{\nu}=\frac{\beta^{[-H, H]}}{\alpha^{[-H, H]}}=\nu^{H} .
$$

Получаем: при $r_{0}>0$

$$
\begin{aligned}
\mathbf{P}\left(\widetilde{X}\left(\widetilde{\kappa}_{0}\right)=r_{0}, \ldots, \widetilde{X}\left(\widetilde{\kappa}_{n}\right)=r_{n}\right)= & C\left(r_{0}\right) \prod_{l=0}^{\lceil(n-1) / 2\rceil-1} p_{\min }\left(r_{2 l+2}-r_{2 l+1}\right) \\
& \times \prod_{l=0}^{\lceil(n-2) / 2\rceil} p_{\max }\left(r_{2 l+1}-r_{2 l}\right),
\end{aligned}
$$


при $r_{0}<0$

$$
\begin{aligned}
\mathbf{P}\left(\widetilde{X}\left(\widetilde{\kappa}_{0}\right)=k_{0}, \ldots, \tilde{X}\left(\widetilde{\kappa}_{n}\right)=k_{n}\right)= & C\left(r_{0}\right) \prod_{l=0}^{\lceil(n-1) / 2\rceil-1} p_{\max }\left(r_{2 l+2}-r_{2 l+1}\right) \\
& \times \prod_{l=0}^{\lceil(n-2) / 2\rceil} p_{\min }\left(r_{2 l+1}-r_{2 l}\right),
\end{aligned}
$$

где

$$
\begin{gathered}
C\left(r_{0}\right)= \begin{cases}\frac{\nu^{H}}{1+\nu^{H}} & \text { при } r_{0}=1, \\
\frac{1}{1+\nu^{H}} & \text { при } r_{0}=-1,\end{cases} \\
p_{\max }(r)=\left(\frac{\nu^{H}}{1+\nu^{H}}\right)^{1+r} \frac{1}{1+\nu^{H}} \mathbf{I}(r \geqslant-1), \\
p_{\min }(r)=\left(\frac{1}{1+\nu^{H}}\right)^{1-r} \frac{\nu^{H}}{1+\nu^{H}} \mathbf{I}(r \leqslant 1),
\end{gathered}
$$

а также

$$
\begin{aligned}
\mathbf{E} \widetilde{\kappa}_{n}= & S_{0}+S_{\max }\left(P_{U}\left\lceil\frac{n}{2}\right\rceil+P_{D}\left\lceil\frac{n-1}{2}\right\rceil\right) \\
& +S_{\min }\left(P_{U}\left\lceil\frac{n-1}{2}\right\rceil+P_{D}\left\lceil\frac{n}{2}\right\rceil\right) \quad \text { при } p \neq q, \\
\mathbf{E} \widetilde{\kappa}_{n}= & 2 n+1 \quad \text { при } p=q,
\end{aligned}
$$

где

$$
\begin{gathered}
S_{0}=\frac{H}{p-q} \frac{\nu^{H}-1}{\nu^{H}+1}, \quad S_{\max }=\frac{H}{p-q}\left(\nu^{H}-1\right), \quad S_{\min }=\frac{H}{p-q}\left(1-\nu^{-H}\right), \\
P_{U}=\frac{\nu^{H}}{1+\nu^{H}}, \quad P_{D}=\frac{1}{1+\nu^{H}} .
\end{gathered}
$$

Подставляя полученные выражения в (3.1), а также пользуясь тем, что согласно следствию 1

$$
\mathbf{E} \tau^{[-H, H]}=\frac{H}{p-q} \frac{\nu^{H}-1}{\nu^{H}+1},
$$

получаем утверждения теоремы.

Для того чтобы вычислить преобразование Лапласа для момента Ренко $\rho_{n}$, рассмотрим разложение

$$
\mathbf{E} e^{-\lambda \rho_{n}}=\mathbf{E} e^{-\lambda \rho_{n}} \mathbf{I}\left(X\left(\widetilde{\rho}_{1}\right)=H\right)+\mathbf{E} e^{-\lambda \rho_{n}} \mathbf{I}\left(X\left(\widetilde{\rho}_{1}\right)=-H\right) .
$$


Как и при доказательстве аналогичного свойства для моментов Каги, индуктивно можно показать, что

$$
\begin{aligned}
& \mathbf{E} e^{-\lambda \rho_{n}} \mathbf{I}\left(X\left(\widetilde{\rho}_{1}\right)=H\right)=\mathbf{E} e^{-\lambda \tilde{\rho}_{1}} \mathbf{I}\left(X\left(\widetilde{\rho}_{1}\right)=H\right)\left(D_{\max }\right)^{\lceil n / 2\rceil}\left(D_{\min }\right)^{\lceil(n-1) / 2\rceil}, \\
& \mathbf{E} e^{-\lambda \rho_{n}} \mathbf{I}\left(X\left(\widetilde{\rho}_{1}\right)=-H\right)=\mathbf{E} e^{-\lambda \tilde{\rho}_{1}} \mathbf{I}\left(X\left(\widetilde{\rho}_{1}\right)=-H\right)\left(D_{\max }\right)^{\lceil(n-1) / 2\rceil}\left(D_{\min }\right)^{\lceil n / 2\rceil},
\end{aligned}
$$

где

$$
D_{\max }=\mathbf{E} \exp \left\{-\lambda \sum_{i=1}^{\tilde{\gamma}_{\max }} \widetilde{\rho}_{i}\right\}, \quad \widetilde{\gamma}_{\max }=\inf \left\{n \geqslant 0: \max _{[0, n]} \tilde{X}-\tilde{X}(n)=H\right\} .
$$

Тогда

$$
\begin{aligned}
& D_{\max }= \sum_{k=1}^{\infty} \mathbf{E} \exp \left\{-\lambda \sum_{i=1}^{k} \widetilde{\rho}_{i}\right\} \mathbf{I}\left(\widetilde{\gamma}_{\max }=k\right) \\
&=\sum_{k=1}^{\infty} \mathbf{E} e^{-\lambda \tilde{\rho}_{1}} \mathbf{I}(\widetilde{X}(1)=H) \cdots e^{-\lambda \tilde{\rho}_{k-1}} \mathbf{I}(\widetilde{X}(k-1)-\widetilde{X}(k-2)=H) \\
& \quad \times e^{-\lambda \tilde{\rho}_{k}} \mathbf{I}(\widetilde{X}(k)-\widetilde{X}(k-1)=-H) \\
&=\sum_{k=1}^{\infty} \mathbf{E} e^{-\lambda \tilde{\rho}_{1}} \mathbf{I}\left(X\left(\widetilde{\rho}_{1}\right)=H\right) \cdots e^{-\lambda \tilde{\rho}_{k-1}} \mathbf{I}\left(X\left(\widetilde{\rho}_{k-1}\right)-X\left(\widetilde{\rho}_{k-2}\right)=H\right) \\
& \quad \times e^{-\lambda \tilde{\rho}_{k}} \mathbf{I}\left(X\left(\widetilde{\rho}_{k}\right)-X\left(\widetilde{\rho}_{k-1}\right)=-H\right) \\
&=\sum_{k=1}^{\infty}\left(\mathbf{E} e^{-\lambda \tilde{\rho}_{1}} \mathbf{I}\left(X\left(\widetilde{\rho}_{1}\right)=H\right)\right)^{k-1} \mathbf{E} e^{-\lambda \tilde{\rho}_{1}} \mathbf{I}\left(X\left(\widetilde{\rho}_{1}\right)=-H\right) \\
&=\frac{\mathbf{E} e^{-\lambda \tilde{\rho}_{1}} \mathbf{I}\left(X\left(\widetilde{\rho}_{1}\right)=-H\right)}{1-\mathbf{E} e^{-\lambda \tilde{\rho}_{1}} \mathbf{I}\left(X\left(\widetilde{\rho}_{1}\right)=H\right)} .
\end{aligned}
$$

Применяя лемму 1 , находим:

$$
\begin{aligned}
D_{\max }= & \operatorname{sh}\left(H \frac{f_{+}(\lambda)+f_{-}(\lambda)}{2}\right) e^{-H\left(f_{-}(\lambda)-f_{+}(\lambda)\right) / 2} \\
\times & {\left[\operatorname{sh}\left((H+1) \frac{f_{+}(\lambda)+f_{-}(\lambda)}{2}\right)\right.} \\
& \left.\quad-\operatorname{sh}\left(H \frac{f_{+}(\lambda)+f_{-}(\lambda)}{2}\right) e^{H\left(f_{-}(\lambda)-f_{+}(\lambda)\right) / 2}\right]^{-1},
\end{aligned}
$$

где $f_{+}(\lambda)=\ln \left(\left(e^{\lambda}+\sqrt{e^{2 \lambda}-4 p q}\right) /(2 p)\right), f_{-}(\lambda)=\ln \left(\left(e^{\lambda}+\sqrt{e^{2 \lambda}-4 p q}\right) /(2 q)\right)$. Аналогично находим

$$
\begin{aligned}
D_{\min }= & \operatorname{sh}\left(H \frac{f_{+}(\lambda)+f_{-}(\lambda)}{2}\right) e^{H\left(f_{-}(\lambda)-f_{+}(\lambda)\right) / 2} \\
\times & {\left[\operatorname{sh}\left((H+1) \frac{f_{+}(\lambda)+f_{-}(\lambda)}{2}\right)\right.} \\
& \left.\quad-\operatorname{sh}\left(H \frac{f_{+}(\lambda)+f_{-}(\lambda)}{2}\right) e^{-H\left(f_{-}(\lambda)-f_{+}(\lambda)\right) / 2}\right]^{-1} .
\end{aligned}
$$


Также из леммы 1 следует, что

$$
\mathbf{E} e^{-\lambda \rho_{1}} \mathbf{I}\left(X\left(\widetilde{\rho}_{1}\right)=H\right)=F_{+}(H, \lambda), \quad \mathbf{E} e^{-\lambda \rho_{1}} \mathbf{I}\left(X\left(\widetilde{\rho}_{1}\right)=-H\right)=F_{-}(H, \lambda),
$$

где

$$
F_{ \pm}(H, \lambda)=\frac{\operatorname{sh}\left(H\left(f_{+}(\lambda)+f_{-}(\lambda)\right) / 2\right) e^{ \pm\left(f_{-}(\lambda)-f_{+}(\lambda)\right) / 2}}{\operatorname{sh}\left((H+1)\left(f_{+}(\lambda)+f_{-}(\lambda)\right) / 2\right)} .
$$

Подставляя (3.9)-(3.12) в (3.11) получаем утверждение теоремы.

4. Торговая стратегия Каги. Рассмотрим применение полученных результатов на примере задачи вычисления прибыли инвестора, который использует стратегию Каги на временном интервале $\left[0, \kappa_{M}\right]$ (см. [2]):

$$
\gamma(t)=\sum_{m=1}^{M} \operatorname{sign}\left(X\left(\kappa_{m-1}\right)-X\left(\kappa_{m-1}^{*}\right)\right) \cdot \mathbf{I}\left(t \in\left[\kappa_{m-1}, \kappa_{m}\right)\right),
$$

где $\gamma(t)$ означает количество единиц актива, которое находится в портфеле инвестора в момент времени $t$, т.е. в момент $\kappa_{n}$ он покупает единицу актива, если $X\left(\kappa_{n}\right)-X\left(\kappa_{n-1}\right)>0$, и продает единицу актива, если $X\left(\kappa_{n}\right)-X\left(\kappa_{n-1}\right)<0$. При этом в каждый момент времени в портфеле инвестора содержится либо 1 единица актива, либо -1 (короткая продажа). Также для простоты будем считать, что период торговли включает в себя ровно $M$ моментов Каги. Прибыль инвестора в этом случае имеет вид

$$
V_{M}=\sum_{m=1}^{M}(-1)^{m+1} \operatorname{sign}\left(X\left(\kappa_{0}\right)\right)\left(X\left(\kappa_{m}\right)-X\left(\kappa_{m-1}\right)\right) .
$$

Тогда ожидаемая прибыль инвестора для случая, когда цена является случайным блужданием, равна

$$
\begin{aligned}
\mathbf{E} V_{M}=(p-q) & \left(S_{\max }\left(\left\lceil\frac{M}{2}\right\rceil P_{U}+\left\lceil\frac{M-1}{2}\right\rceil P_{D}\right)\right. \\
& \left.-S_{\min }\left(\left\lceil\frac{M-1}{2}\right\rceil P_{U}+\left\lceil\frac{M}{2}\right\rceil P_{D}\right)\right),
\end{aligned}
$$

где величины $P_{U}, P_{D}, S_{\max }, S_{\min }$ даны в $(2.22)$ и $(2.25)$ соответственно.

Чтобы это показать, рассмотрим введенные в $(2.21)$ события $U=$ $\left\{X\left(\kappa_{0}\right)>0\right\}$ и $D=\left\{X\left(\kappa_{0}\right)<0\right\}$. Тогда имеет место следующее представление:

$$
V_{M}=V_{M}^{+} \mathbf{I}(U)+V_{M}^{-} \mathbf{I}(D),
$$


где

$$
\begin{aligned}
& V_{M}^{+}=\sum_{m=1}^{M}(-1)^{m+1}\left(X\left(\kappa_{m}\right)-X\left(\kappa_{m-1}\right)\right), \\
& V_{M}^{-}=\sum_{m=1}^{M}(-1)^{m}\left(X\left(\kappa_{m}\right)-X\left(\kappa_{m-1}\right)\right) .
\end{aligned}
$$

Для случая $p \neq q$ из теоремы 1 получаем, что

$$
\begin{aligned}
& \mathbf{E} V_{M}^{+} \mathbf{I}(U)=P_{U}(p-q)\left(S_{\max }\left\lceil\frac{M}{2}\right\rceil-S_{\min }\left\lceil\frac{M-1}{2}\right\rceil\right), \\
& \mathbf{E} V_{M}^{-} \mathbf{I}(D)=P_{D}(p-q)\left(S_{\max }\left\lceil\frac{M-1}{2}\right\rceil-S_{\min }\left\lceil\frac{M}{2}\right\rceil\right) .
\end{aligned}
$$

Отсюда получаем выражение для ожидаемой прибыли инвестора (4.1). Отметим, что в случае симметричного случайного блуждания $(p=q)$ имеем

$$
\mathbf{E}\left(X\left(\kappa_{m}\right)-X\left(\kappa_{m-1}\right)\right)=0,
$$

поскольку $X$ в этом случае является мартингалом, выходящим из нуля п.н. В этом случае величины $X\left(\kappa_{m}\right)-X\left(\kappa_{m-1}\right)$ не зависят от $X\left(\kappa_{0}\right)$, и, следовательно, ожидаемая прибыль инвестора в симметричном случае равна нулю, что соответствует ситуации с отсутствием арбитража на рынке.

Теперь исследуем вопрос о том, какую ожидаемую прибыль получит инвестор от использования стратегии Каги на бесконечном временном интервале в случае $p \neq q$. Заметим, что для произвольного $n \geqslant 1$ имеет место представление

$$
\kappa_{n}=\sum_{m=0}^{n}\left(\kappa_{m}-\kappa_{m-1}\right)
$$

где $\kappa_{-1}=0$ п.н. Из определения моментов остановки $\kappa_{i}$ нетрудно заметить, что для любого $m \geqslant 1$

$$
\mathbf{P}\left(\kappa_{m}-\kappa_{m-1} \geqslant H\right)=1 .
$$

Следовательно, $\kappa_{n} \uparrow \infty$ п.н. при $n \rightarrow \infty$. Значит, прибыль инвестора на бесконечном временном интервале можно полагать равной $\lim _{M \rightarrow \infty} \mathbf{E} V_{M}$.

Рассмотрим сначала подпоследовательность $M=2 N, N \in \mathbf{N}$. Tогда из $(4.1),(2.22)$ и $(2.25)$ имеем

$$
\mathbf{E} V_{2 N}=N \frac{-\nu^{2 H+1}+(2 H+1) \nu^{H+1}-(2 H+1) \nu^{H}+1}{\nu^{H}(1-\nu)} .
$$

Пусть $f(\nu)$ обозначает многочлен, стоящий в числителе:

$$
f(\nu)=-\nu^{2 H+1}+(2 H+1) \nu^{H+1}-(2 H+1) \nu^{H}+1, \quad \nu \in(0, \infty) .
$$


Тогда

$$
f^{\prime}(\nu)=\nu^{H-1}\left[-(2 H+1) \nu^{H+1}+(2 H+1)(H+1) \nu-(2 H+1) H\right] .
$$

Выражение в квадратных скобках обозначим $g(\nu)$. Функция $g$ достигает своего максимума в точке $\nu=1$, поскольку

$g^{\prime}(\nu)=0 \quad \Leftrightarrow \quad-(2 H+1)(H+1) \nu^{H}+(2 H+1)(H+1)=0 \quad \Leftrightarrow \quad \nu=1$.

Значит, $g(\nu) \leqslant g(1)=0$ для любого $\nu$. Следовательно, $f^{\prime}(\nu) \leqslant 0$ для любого $\nu$, откуда получается, что функция $f$ убывает на $(0, \infty)$. Учитывая равенства $f(0)=1, f(1)=0$, находим, что

$$
f(\nu)>0 \quad \text { при } \nu \in(0,1), \quad f(\nu)<0 \quad \text { при } \nu \in(1, \infty) .
$$

Тогда из (4.2) несложно вывести следующее утверждение:

$$
\lim _{N \rightarrow \infty} \mathbf{E} V_{2 N}= \begin{cases}\infty & \text { при } \nu \neq 1, \\ 0 & \text { при } \nu=1 .\end{cases}
$$

Иными словами, в ситуации, когда на рынке присутствует арбитраж $(p \neq q)$, инвестор, используя указанную выше стратегию Каги, всегда может воспользоваться арбитражной возможностью и получить прибыль.

Если $M=2 N+1, N \in \mathbf{N}$, то имеем следующее представление:

$$
\begin{aligned}
\mathbf{E} V_{2 N+1}= & N \frac{-\nu^{2 H+1}+(2 H+1) \nu^{H+1}-(2 H+1) \nu^{H}+1}{\nu^{H}(1-\nu)} \\
& +\left(P_{U}\left(\frac{\nu-\nu^{H+1}}{1-\nu}-H\right)-P_{D}\left(H-\frac{1-\nu^{H}}{\nu^{H}-\nu^{H+1}}\right)\right) .
\end{aligned}
$$

Второе слагаемое не зависит от $N$, поэтому ответ (4.3) остается прежним.

\section{СПИСОК ЛИТЕРАТУРЫ}

1. Булинский A.В., Ширяев А.Н. Теория случайных процессов. М.: Физматлит, 2005, $402 \mathrm{c}$.

2. Пастухов С.В. О некоторых вероятностно-статистических методах в техническом анализе. - Теория вероятн. и ее примен., 2004, т. 49, в. 2, с. 297-316.

3. Феллер В. Введение в теорию вероятностей и ее приложения. М.: Мир, 1964, 498 c.

4. Ширяев А.Н. О мартингальных методах в задачах о пересечении границ броуновским движением. М.: МИАН, 2007, 78 с. (Совр. пробл. матем., в. 8.)

5. Нисон C. За гранью японских свечей: новые японские методы графического анализа. М.: Евро, 2009, 304 с. 